\title{
A CONDITION EQUIVALENT TO THE SOLVABILITY OF THE ENDOMORPHISM PROBLEM FOR FREE GROUPS
}

\author{
CHARLES C. EDMUNDS ${ }^{1}$
}

\begin{abstract}
To solve the endomorphism problem for a word $\boldsymbol{W}$ involving $\boldsymbol{n}$ generators of a free group $F$, it suffices to solve the problem assuming that the images of $W$ involve no more than $n$ generators.
\end{abstract}

1. Introduction. Let $F$ be the free group freely generated by $x_{1}, x_{2}, \ldots$ The endomorphism problem for the pair $(W, U) \in F \times F$ is to determine whether there is an endomorphism of $F$ sending $W$ to $U$. The endomorphism problem for a set $P$ of pairs is said to be solvable if there is an effective algorithm which solves the endomorphism problem for every $(W, U) \in P$. The endomorphism problem for a word $W$ is obtained by setting $P=\{W\}$ $\times F$.

In this note we will show that it suffices to consider only those words $U$ containing no more distinct generators than occur in $W$. Letting $g(W)$ be the cardinality of the set $\left\{x_{i}: x_{i}\right.$ or $x_{i}^{-1}$ occurs in $\left.W\right\}$, our condition can be stated more concisely as follows:

THEOREM. The endomorphism problem is solvable for $W$ if and only if it is solvable restricted to the set of pairs $\{(W, U): g(U) \leqslant g(W)\}$.

The possibility of such a reduction was suggested to the author by M. J. Wicks (written communication). The reader is referred to [1] for a discussion of definitions and notation.

2. Proof of the theorem. The theorem follows from a combination of certain results in [1]. The necessity of the condition is clear, thus it remains to establish its sufficiency.

To solve the endomorphism problem for a pair $(W, U)$ it is enough, by Whitehead's theorem [3], to solve the problem for some pair $\left(W^{\prime}, U^{\prime}\right)$ where $W^{\prime}$ and $U^{\prime}$ are automorphic images of $W$ and $U$, respectively. Therefore, as in [1], we can assume that $W$ and $U$ are reduced, irredundant, cyclic words. By Theorem 2.1 of [1], $U$ is an endomorphic image of $W$ if and only if $U$ is a $c$-free image of some word in $D_{W}$. Letting $U_{1}, U_{2}, \ldots, U_{n}$ be the distinct (cyclic) words having $U$ as a $c$-free image, it follows that $U$ is an endomorphic image of $W$ if and only if some $U_{1}$ is in $D_{W}$.

Received by the editors October 6, 1978.

AMS (MOS) subject classifications (1970). Primary 20E05; Secondary 20F10, 20 F55.

Key words and phrases. Free group, endomorphism, decision problem.

${ }^{1}$ Research supported by the National Research Council of Canada. 
Our result is trivial if $W$ is universal. If $W$ is nonuniversal then so is every word in $D_{W}$, therefore we can omit any universal $U_{i}$ from further consideration. Lemma 5.2 of [1] implies that for each $U_{i}$ there is a connected word $V_{i}$, effectively calculable from $U_{i}$, which is an automorphic image of $U_{i}$ with $\Delta\left(U_{i}\right) \geqslant \Delta\left(V_{i}\right)$. Clearly $U$ is an endomorphic image of $W$ if and only if some $V_{i}$ is an endomorphic image of $W$. If $U_{i} \in D_{W}$, Proposition 3.8 of [1] implies that $\Delta(W) \geqslant \Delta\left(U_{i}\right)$. Thus $\Delta(W) \geqslant \Delta\left(U_{i}\right) \geqslant \Delta\left(V_{i}\right)=g\left(V_{i}\right)-1$. It follows easily that $g(W) \geqslant g\left(V_{i}\right)$. Assuming then that the endomorphism problem is solvable for the set of pairs $(W, V)$ with $g(V)<g(W)$ it follows that it is solvable for $W$.

REMARK. Note that we have proved the somewhat sharper result that the endomorphism problem is solvable for $W$ if and only if it is solvable for the set of pairs $(W, U)$ with $U$ connected and $\Delta(U) \leqslant \Delta(W)$.

COROLlaRY. Solving the endomorphism problem for a word $W$ in $F$, with $g(W)=n$, is equivalent to solving the endomorphism problem for $W$ in $F_{n}$ $\left(=\left\langle x_{1}, x_{2}, \ldots, x_{n} ; \varnothing\right\rangle\right)$.

REMARK. In [2] Paul Schupp solves the endomorphism problem for $W$ any two-generator word in $F_{n}(n \geqslant 2)$. This is equivalent to solving the endomorphism problem for $F_{2}$ (i.e., for every word in $F_{2}$ ).

The three-generator case is open. Here our result shows that, instead of studying the images of three-generator words in $F$, it is enough to solve the endomorphism problem for $F_{3}$.

\section{REFERENCES}

1. C. C. Edmunds, On the endomorphism problem for free groups. II, Proc. London Math. Soc. 38 (1979), 153-168.

2. P. E. Schupp, On the substitution problem for free groups, Proc. Amer. Math. Soc. 23 (1969), 421-424.

3. J. H. C. Whitehead, On certain sets of elements in a free group, Proc. London Math. Soc. 41 (1936), 48-56.

Department of Mathematics, Mount Saint Vincent University, Halifax, Nova Scotia, Canada 